\title{
Color Thresholding Method for Image Segmentation of Natural Images
}

\author{
Nilima Kulkarni \\ New Horizon College of Engineering, Bangalore, India \\ e-mail: kulkarninilima@gmail.com
}

\begin{abstract}
Most of the thresholding procedures involved setting of boundaries based on grey values or intensities of image pixels. In this paper, the thresholding is to be done based on color values in natural images. The color thresholding technique is being carried out based on the adaptation and slight modification of the grey level thresholding algorithm. Multilevel thresholding has been conducted to the RGB color information of the object extract it from the background and other objects. Different natural images have been used in the study of color information. The results showed that by using the selected threshold values, the image segmentation technique has been able to separate the object from the background.
\end{abstract}

Index Terms-Color image segmentation, Color thresholding, Multilevel thresholding, Natural images, RGB color information.

\section{INTRODUCTION}

Segmentation process subdivides an image into its constituent regions or objects. The level of subdivision depends on the problem being solved, where the segmentation should stop when the objects of interest in an application have been isolated.

Image segmentation refers to partitioning of an image into different regions that are homogeneous or "similar" in some image characteristics. It is usually the first task of any image analysis process module and thus, subsequent tasks rely strongly on the quality of segmentation [10]. Various techniques have been proposed in the literature where color, edges, and texture were used as properties for segmentation. Using these properties, images can be analyzed for use in several applications including video surveillance, image retrieval, medical imaging analysis, and object classification.

On the outset, segmentation algorithms were implemented using grayscale information only [2]. The advancement in color technology facilitated the achievement of meaningful segmentation of images as described in [3, 4]. The use of color information can significantly improve discrimination and recognition capability over gray-level methods.

However, early procedures consisted of clustering pixels by utilizing only color similarity. Spatial locations and correlations of pixels were not taken into account yielding, fragmented regions throughout the image. Statistical methods, such as Classical Bayes decision theory, which are based on previous observation, have also been quite popular [5, 6]. However, these methods depend on global a priori knowledge about the image content and organization. Until recently, very little work had used underlying physical models of the color image formation process in developing color difference metrics.

Because the human eyes have adjustability for the brightness, which we can only identified dozens of Gray-scale at any point of complex image, but can identify thousands of colors. In many cases, only utilize gray-Level information cannot extract the target from background; we must by means of color information. Accordingly, with the rapidly improvement of computer processing capabilities, the color image processing is being more and more concerned by people $[25,31]$. The color image segmentation is also widely used in many multimedia applications, for example; in order to effectively scan large numbers of images and video data in digital libraries, they all need to be compiled directory, sorting and storage, the color and texture are two most important features of information retrieval based on its content in the images and video. Therefore, the color and texture segmentation often used for indexing and management of data; another example of multimedia applications is the dissemination of information in the network [26]. Today, a large number of multimedia data streams sent on the Internet, However, due to the bandwidth limitations; we need to compress the data, and therefore it calls for image and video segmentation.

Human eyes can distinguish thousands of colors but can only distinguish 20 kinds of grayscale, so we can easily and accurately find the target from the color images. However, it is difficult to find out from the gray-scale image. The reason is that color can provide more information than grayscale. The color for the pattern recognition and machine vision is very useful and necessary [27]. At present, specifically applied to the color image segmentation approach is not so much as for the gray-scale images, most of proposed color image segmentation methods are the combination of the existing grayscale image segmentation method on the basis of different color space. Commonly used for color image segmentation methods are histogram threshold, feature space clustering, region-based approach, based on edge detection methods, fuzzy methods, artificial 
neural network approach, based on physical model methods, etc.

Another challenging aspect of image segmentation is the extraction of perceptually relevant information. Since humans are the ultimate users of most CBIR systems, it is important to obtain segmentations that can be used to organize image contents semantically, according to categories that are meaningful to humans. This requires the extraction of low-level image features that can be correlated with high-level image semantics. However, rather than trying to obtain a complete and detailed description of every object in the scene, it may be sufficient to isolate certain regions of perceptual significance (such as "sky," "water," "mountains," etc.) that can be used to correctly classify an image into a given category, such as "natural," "man-made," "outdoor," etc. [28].

The different methods of image segmentation algorithms for images

- Edge based image segmentation method

- Adaptive thresolding method

- Watershed method

- Region growing by active contour method

- Quadtree method

- Fuzzy c means clustering method

Edge based image segmentation method : In the first step, the canny edge detector is used to process the two parameter images and then the derived edges are added to derive the final edge detection results. After that local thresholding technique is applied. Adaptive thresholding method: Thresholding is called adaptive thresholding when different thresholds are used for different regions in the image [29]. This may also be known as local or dynamic thresholding. Consider a grayscale document image in which $\mathrm{g}(\mathrm{x}, \mathrm{y}) €[0,255]$ be the intensity of a pixel at location $(\mathrm{x}, \mathrm{y})$. In local adaptive thresholding techniques, the aim is to compute a threshold $t(x, y)$ for each pixel such that

$$
O(x, y)=\left\{\begin{array}{l}
0, . i f g(x, y) \leq t(x, y) \\
255, \text { otherwise }
\end{array}\right.
$$

Watershed segmentation method: In image watershed segmentation, altitude is represented by the gray level of the pixels. All pixels throughout the same catchment basin are connected with the minimum altitude region of the basin [1]. The watershed lines divide individual catchment basins. The high gradient regions correspond to watershed lines and low gradient regions correspond to catchment basins. Region Growing by active contour method: Region-based active contour method is to find the image partition that minimizes a criterion including both region-based and boundary-based terms. Each region is described by a function named "descriptor" of the region. First, for a given application like detection of moving objects, various descriptors can be easily tested inside the same theoretical framework. Second, this framework can be applied to other applications [30].

Quad tree method: The QT structure allows to divide an image within a complete tree representation, including neighboring information. This spatial information can be further used by a merging strategy which joins the QT leaves using color and edge information. Fuzzy c means clustering: The purpose of clustering is to identify natural groupings of data from a large data set to produce a concise representation of a system's behavior. Fuzzy c-means (fcm) is a data clustering technique in which a dataset is grouped into $\mathrm{n}$ clusters with every data point in the dataset belonging to every cluster to a certain degree $[34,35]$.

By regarding the image segmentation as a problem of partitioning pixels into different clusters according to their color similarity and spatial relation, we propose our color image segmentation method automatically.

Image segmentation algorithms generally are based on one of the two basic properties of intensity values: discontinuity and similarity. Thresholding is a method of similarity category. It partitions an image into regions that are similar according to a set of predefined criteria. There are various thresholding techniques and it is also a fundamental approach to segmentation that enjoys a significant degree of popularity, especially in applications where speed is an important factor [17].

In this paper, a method of grey thresholding technique is reviewed, and it is then being adapted to suit with color images.

The remainder of the paper is organized as follows. In section II, a review of the gray thresholding technique is presented. The proposed algorithm of multilevel thresholding for natural images described in Section III. After that, application of the proposed algorithm is discussed in section IV, and we draw our conclusion in the last section.

\section{GRAY THRESHOLDING}

Traditionally, one simple way to accomplish thresholding is by defining a range of brightness value in the original image, then selects the pixels within the range as belonging to the foreground and rejects all of other pixels to the background. Such an image is then usually displayed as a binary or two-level image [18]. The general rule for grey level pixel thresholding is as follows 


$$
g(x, y)= \begin{cases}0, & f(x, y)<T, \\ 1, & f(x, y) \geq T\end{cases}
$$

where $T$ is the threshold value, $f(x, y)$ is the original pixel value, and $\mathrm{g}(\mathrm{x}, \mathrm{y})$ is the resulted pixel value after thresholding has been done. Equation 1 specifies 0 and 1 as output values, which will give the result as a true binary image. Equation 1 can be further visualized by Figure 1 as mappings of input grey level to output grey level [19].

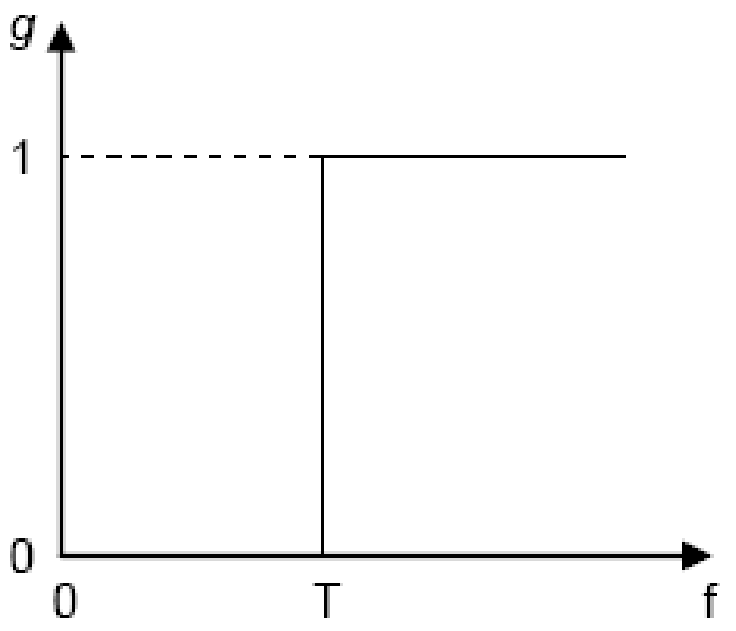

Fig. 1. Thresholding for a single threshold

There could be more than one thresholding value at a time, which change Equation 1, to

$$
g(x, y)= \begin{cases}0, & f(x, y)<T_{1}, \\ 1, & T_{1} \leq f(x, y) \leq T_{2}, \\ 0, & f(x, y)>T_{2} .\end{cases}
$$

Where $T_{1}$ is the lower threshold value and $T_{2}$ is the upper threshold value. Figure 2 shows the visualization of how thresholding with a pair of threshold is being done [20].

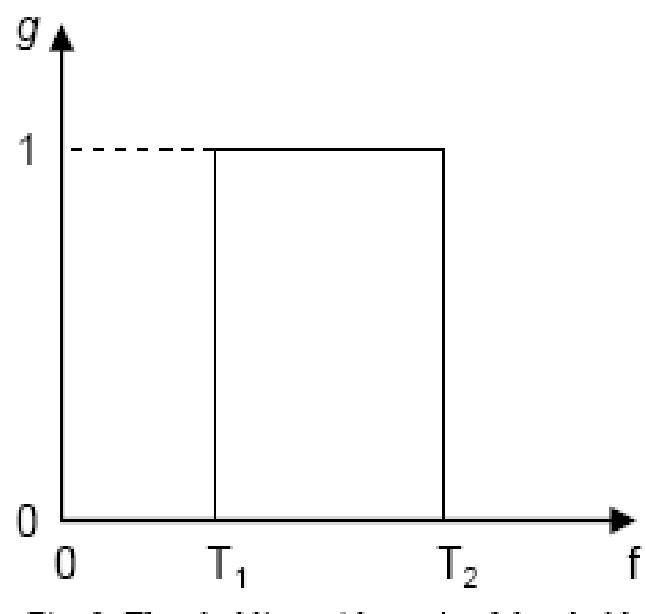

Fig. 2. Thresholding with a pair of threshold

For color images, more than one variable characterizes each pixel in the image, which allows multi spectral thresholding [21]. In color imaging, each pixel is characterized by three RGB values. However, with multi spectral or multilayer images such as RGB model, it can be difficult to specify the selection criteria. The logical extension of thresholding is simply to place brightness thresholds on each image, for instance to specify the range of red, blue and green intensities.

These multiple criteria are then usually combined with an AND operation (i.e. the pixel is defined as part of the foreground if its three RGB components all lie within the selected range). This logically equivalent to segmenting each image plane individually, creating separate binary images and then combining them with a Boolean AND operator afterward. This color thresholding method is widely used in the image segmentation [20, 22, 23and 24].

\section{PROPOSED MULTILEVEL THRESHOLDING FOR NATURAL IMAGES}

A brief study on the color information of the natural images was carried out in order to get the most suitable values for selection range of the threshold. The study was carried out on different types of natural images, which comprise of normal images, low quality images, compressed images. The color thresholding technique was carried out based on the color information of the object to extract it images from the background and other objects. This technique specifies the range of RGB intensities for thresholding. The objects that lie outside the selection range will be rejected. Therefore, it is very important to determine the selection range because if this threshold cannot acquire a suitable value, the thresholding algorithm will extract pixels other than the expected object.

The properties of the RGB pixels are being studied to extract the important features from the image, for example, if we are interested in green areas (called as 
Forest) then based on the color information, the color thresholding algorithm should be able to extract the pixels of green color and reject pixels of other objects. If we are interested in blue areas (called as $S k y$ ) then color thresholding algorithm should able to extract blue color and reject pixels of other objects. Following are the steps for proposed approach

\section{Original Image}

\section{Calculate range of \\ RGB intensities for thresholding}

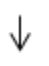

\section{Apply
thresholding}

Fig. 3 Steps for RGB Thresholding algorithm

In this paper, we are dealing with the natural images and we are interested in green areas (called as Forest) and blue areas (called as Sky). We calculated range for RGB intensities for green and blue colors, and then we apply thresholding algorithm.

In order to view the important properties of each segment so that necessary features and accurate value of threshold can be obtained from the result, the information is being gathered in a table. In this table, among the features that are noted are the maximum and minimum values for each of the RGB components in green area and blue area respectively. The maximum and minimum values for each of the pixels are also noted to extract important characteristic of the RGB values that may be converted into threshold values. Summary of the findings from the study can be visualized in Table 1 and Table 2.

TABLE 1 : RGB INFORMATION IN GREEN AREA

\begin{tabular}{|c|c|c|}
\hline & MIN & MAX \\
\hline RED & 0 & 173 \\
\hline GREEN & 102 & 255 \\
\hline BLUE & 0 & 173 \\
\hline
\end{tabular}

TABLE 2 : RGB INFORMATION IN BLUE AREA

\begin{tabular}{|c|c|c|}
\hline & MIN & MAX \\
\hline RED & 0 & 90 \\
\hline BLUE & 127 & 255 \\
\hline GREEN & 127 & 255 \\
\hline
\end{tabular}

From the data in the table, it can be seen that there are various combination of values that can be used to determine the best threshold for this type of image. Since the determination of the best result can only be done by human observation, various attempts have been done so that the results can be compared to select the best values for the thresholding algorithm.

Among the combinations that have been considered were the ranges of minimum and maximum values for each of the RGB components, the average values, as well as the obvious difference between each of the RGB components.

Considering the minimum and maximum values of RGB components, Equations (3), (4), and (5) have been formulated to get the thresholding values for green color (i.e. forest). However, the original values have been modified to cater up to $10 \%$ of difference.

$$
\begin{aligned}
& g(x, y)= \begin{cases}f(x, y), & 0 \leq \operatorname{red}(x, y) \leq T r, \\
g 1(x, y), & \text { red }(x, y)>T r .\end{cases} \\
& g(x, y)= \begin{cases}f(x, y), & \text { Tg } \leq \operatorname{green}(x, y) \leq 255, \\
g 1(x, y), & \text { green }(x, y)<T g .\end{cases} \\
& g(x, y)= \begin{cases}f(x, y), & 0 \leq \text { blue }(x, y) \leq T b, \\
g 1(x, y), & \text { blue }(x, y)>T b\end{cases}
\end{aligned}
$$

where $\mathrm{g} 1(\mathrm{x}, \mathrm{y})$ is the gray value of pixel and $\operatorname{red}(\mathrm{x}, \mathrm{y})$, $\operatorname{green}(\mathrm{x}, \mathrm{y})$ and blue $(\mathrm{x}, \mathrm{y})$ are the pixel values for each of the red, green and blue components respectively.

From Equations (3), (4), and (5), it can be seen that the original equation that has been mentioned in Equations (1) and (2) have been slightly modified to adopt the method of grey level thresholding to color thresholding. For the new equations, each RGB component is being treated independently. Since there are three components, the thresholding process is being done to one component at a time, and they are then combined into 1 rule using a Boolean AND operator.

Another modification that has been made is that, the output value is not 0 or 1 , but either 255 (white pixel) or retaining the old value of the pixel. This means that if the value of that particular pixel falls in the range of the rule whereby the output value is original pixel color, this indicates object we are interested in. However, if it is not fall within that range the gray value of the pixel is retained.

Equations (6), (7), and (8) have been formulated to get the thresholding values for blue (i.e. Sky) color. 


$$
\begin{aligned}
& g(x, y)= \begin{cases}f(x, y), \quad 0 \leq \text { red }(x, y) \leq T r, \\
g 1(x, y), & \text { red }(x, y)>T r .\end{cases} \\
& g(x, y)= \begin{cases}f(x, y), \quad T g \leq \text { green }(x, y) \leq 255, \\
g 1(x, y), & \text { green }(x, y)<T g .\end{cases} \\
& g(x, y)= \begin{cases}f(x, y), & \text { Tb } \leq \text { blue }(x, y) \leq 255, \\
g 1(x, y), & \text { blue }(x, y)<T b\end{cases}
\end{aligned}
$$

It is observed from the experiments that, threshold value $\mathrm{Tr}=173, \mathrm{Tg}=102$ and $\mathrm{Tb}=173$ gives best results for segmentation of green area (i.e. forest) in natural image. And threshold value $\mathrm{Tr}=90, \mathrm{Tg}=127$ and $\mathrm{Tb}$ $=127$ gives best results for segmentation of blue area (i.e. sky) in natural image.

In order to determine whether the thresholding method that has been carried out is successful or not, it relies solely on human intervention. Therefore, the threshold value need to be varied until acceptable results are achieved, based on the human observation. That is why, in carrying the color thresholding procedure, it may be necessary to do a few level of thresholding in order to get the best results.

The thresholding procedure must be done to the red, green and blue components. The thresholding on the three colors may be combined into one complete rule using the Boolean AND operator or it may also be separated into two or more rules.

If more than one rule is being created, then it is considered to be done on a few level of thresholding. In this paper, the process of thresholding is being done automatically based on the predetermined threshold value.

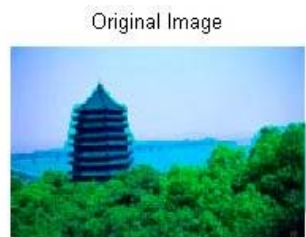

(a)

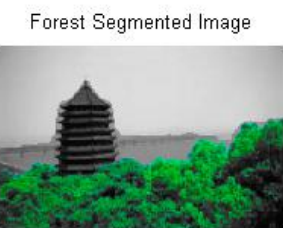

(b)

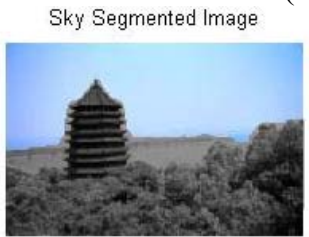

(c)

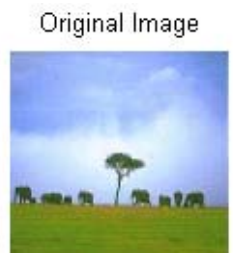

(d)

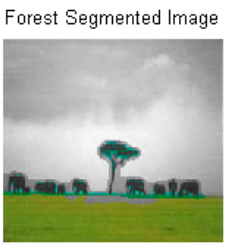

(e)

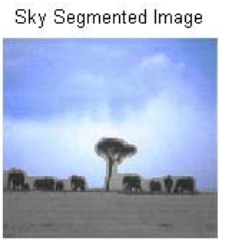

(f)

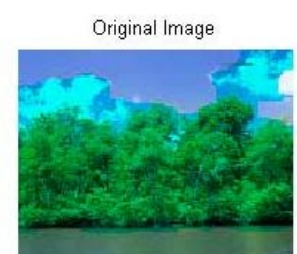

(g)

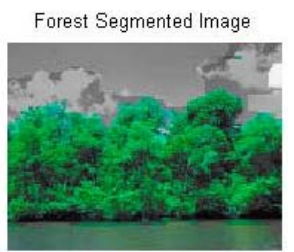

(h)

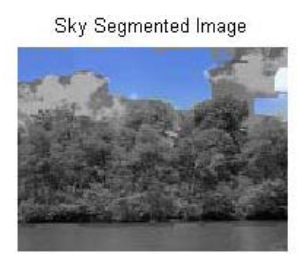

(i)

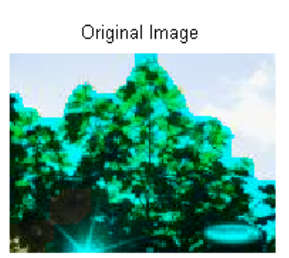

(j)

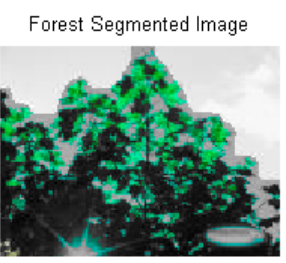

(K)

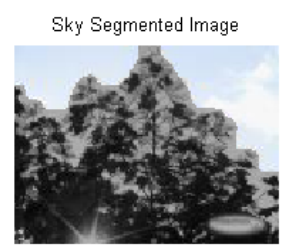

(1)

Fig. 4 figures (a), (d), (g) and (j) are the original images Figures (b), (e), (h) and (k) are the result of green color segmentation and Figures (c), (f), (i ) and (l) are the result of blue color segmentation. 
Fig (a), (d) and (g) shows original image. If we are interested in green color segments (or forest), then we can apply the thresholding values as in equation (3),(4) and (5). The results are shown in fig (b),(e) and (h). If we are interested in blue color segments (or sky), then we can apply the thresholding values as in equation (6),(7) and (8). The results are shown in fig (c),(f) and (i). The proposed algorithm is applied on many natural images and experimental results shows that it provides simple and effective color image segmentation.

\section{CONCLUSION}

A technique of image segmentation by conducting a thresholding method for natural images has been presented. The segmentation allows the elimination of a great amount of unwanted pixels, and retained only those pixels in object we are interested in. The resulted images satisfactorily showed that by using the selected threshold values, the image segmentation method has been able to segment out the green area (i.e. forest) and blue area (i.e. sky) from natural images.

The meaningful experiment results of color image segmentation hold favorable consistency in terms of human perception and satisfy the content-based image retrieval and recognition process. There is one disadvantage in proposed algorithm. Although using the fixed threshold values can produce reasonably good results, it may not generate the best results for all the images. However this proposed thresholding algorithm can be combine with other properties such as texture into the algorithm to improve segmentation performance is the point of our further research.

The performance of the proposed algorithms has been demonstrated in the domain of photographic images, including low resolution, degraded, and compressed images.

\section{REFERENCES}

[1] ZHANG YU-JIN. Image project (media), image analysis. Beijing. Tsinghua University Press, 2005

[2] H. Cheng, X. Jiang, Y. Sun and J. Wang, Color image segmentation: Advances \& prospects, Pat. Rec., Vol. 34, No. 12, pp. 2259-2281, Dec. 2001

[3] J. Wu, H. Yan, and A. Chalmers, "Color image segmentation using fuzzy clustering and supervised learning", Journal of Elec. Imag., Vol. 3, No. 4, pp. 397-403, Oct. 1994.

[4] P. Schmid, Segmentation of digitized dermatoscopic images by two-dimensional color clustering, IEEE Trans. on Med. Image., Vol. 18, No.2, pp. 164-171, Feb. 1999.

[5] Daily, M.J., J.G. Harris, K.E. Olin, K. Reiser, D.Y. Tseng, and F.M. Vilnrotter, Knowledge-based Vision Techniques Annual Technical Report. U.S. Army ETL, Fort Belvoir, VA, October, 1987.

[6] Healey, G. and T. Binford, The Role and Use of Color in a General Vision System. Proc. of the DARPA IU Workshop, Los Angeles, CA, pp. 599-613, February, 1987.

[7] GONG Sheng-rong, Digital image processing and analysis. Beijing. Tsinghua Unversity Press, 2005

[8] G.Wyszecki and W.Stiles, Color Science: Concepts and Metheds, Quantitative Data and Formulae, 2nd ed. New York: Wiley, 1982.

[9] H.D. Cheng, X.H. Jiang, Y. Sun, et al. "Color image segmentation: advances and prospects". Pattern Recognition, 2001, pp. 22592281

[10] Qi Yonghong and Zhou Shshenqi, "Review on Uniform color space and Color Difference Formula", Print World, 2003.9:16-19.

[11] Gong Y.H, Proietti G. Image indexing and retrieval based on human perceptual color clustering. The international conference on computer vision, Munbai, 1998

[12] S. Wesolkowski, M.E. Jernigan, R.D. Dony, "Comparison of color image edge detectors in multiple color space". ICIP-2000, pp. $796-799$

[13] J. Maeda, V.V.Anh,T.Ishizaka and y. suzuki, “ Integration of local fractal dimension and boundary edge in segmenting natural images" , Proc. IEEE Int. Conf. on Image Processing, vol.I, pp.845-848, 1996.

[14] J. Maeda, T. Lizawa, T. Ishizaka, C. Ishikawa and Y. Suzuki, "Segmentation diffusion and linking of boundary edges", Pattern Recognition, vol.31(12), pp.1993-1999, 1998.

[15] Ye Qixiang, Gao Wen, Wang Weiqiang, Hang Tiejun. “A Color Image Segmentation Algorithm by Using Color and Spatial Information". Journal of Software. 2004, 15(4):522-530.

[16] Hsin-Chia Chen, Sheng-Jyh Wang. "The use of visible color difference in the quantitative evaluation of color image segmentation". Vision, image and signal processing, IEEE proceedings. 2006, Vol.153, pp.598 - 609.

[17] R. C. Gonzalez, and R. E. Woods, Digital Image Processing, Second Edition. Pearson Education, 2nd Ed., New Jersey, 2002.

[18] M. Sezgin and B. Sankur, "Survey over image thresholding techniques and quantitative performance evaluation," Journal of Electronic Imaging, Vol. 13, pp. 146-165, 2004.

[19] N. Efford, Digital Image Processing: a practical introduction using Java. Pearson Education, USA, 2000.

[20] M. G. Forero, F. Sroubek, and G. Cristobal. "Identification of tuberculosis bacteria based on shape and color," Real-Time Imaging Vol. 10, pp. 251-262, Aug. 2004.

[21] N. Otsu, "A threshold selection method from gray level histograms.," IEEE Transactions on Systems, Man and Cybernetics, Vol. 9, No. 1, pp. 62-66, 1979.

[22] M. G. Forero, G. Cristobal, and J. A. Borrego, "Automatic identification techniques of tuberculosis bacteria," in: SPIE Proceedings Of The Applications Of Digital Image Processing XXVI, Vol. 5203, pp. 71-81, 2003.

[23] C. Mancas-Thillou, and B. Gosselin, "Color binarization for complex camera-based images", Proc. of the Electronic Imaging Conference of the International Society for Optical Imaging (SPIE/IS\&T), San Jose (California, USA), 2005.

[24] Liu He. Digital Image Processing and Application. Beijing: China Electric Power Press, 2006.

[25] Peck, M.ACell Image Segmentation of Gastric Cancer Based on Region-Growing and Watersheds.. 2002. Quebec City, Que.: Univelt Inc.

[26] Ahmed, J., V.T. Coppola, and D.S. Bernstein, Segmentation of Blood Cells Image Based on Support Vector Machines Control, and Dynamics, 1998.21(5): p. 684-691.

[27] Wilson, E., C. Lages, and R. Mah. Improved research for overlapping segmentation based on watershed algorithm. 2002. Tulsa, OK, United States: Institute of Electrical and Electronics Engineers Inc.

[28] A. Mojsilovic and B. Rogowitz, —Capturing image semantics with low- level descriptors, $\|$ in Proc. Int. Conf. Image Processing, Thessaloniki, Greece, Oct. 2001, pp. 18-21.

[29] Faisal Shafaita, Daniel Keysersa, Thomas M. Breuelb, "Efficient Implementation of Local Adaptive ThresholdingTechniques Using Integral Images", Proceedings of SPIE, 2008.

[30] Tony F. Chan, Member, IEEE, and Luminita A Vese, "Active Contours Without Edges" IEEE Transaction on Image Processing, pp.267-277,No.2, VoLlO, Feb 2001

[31] Chen Wufan. Wavelet Analysis and Its Application on Image Processing. Beijing:Science Press, 2002.

[32] Huang Daren, Bi Lin, Sun Xin. Multi-band Wavelets Analysis. Hangzhou: Zhejiang University Press, 2001.

[33] Sharon E. Hierarchy and Adaptivity in Segmenting Visual Scenes. Nature, 2006, 442(17):810-813. 
[34] Zhang Aihua. The Research of Image Segmentation Based on Fuzzy Clustering [D]. Wuhan: A Dissertation of Huazhong University of Science and Technology, 2004, 1-14.

[35] Zhou Liping, Gao Xinbo. Image Segmentation via Fast Fuzzy CMeans Clustering $[\mathrm{J}]$. Computer Engineering and Application, 2004, 40(8):68-70.

Nilima Kulkarni, female, is a master in engineering (computer science and engineering) working as Asst. Professor in New Horizon College of Engineering, Bangalore, and Country India. Her research interests include digital image processing, image segmentation and computer vision. Her teaching interests include digital image processing, ObjectOriented programming, and operating systems. 